\title{
Computation of Partially Inflated Shapes of Stratospheric Balloon Structures
}

\author{
X. Deng* and S. Pellegrino ${ }^{\dagger}$ \\ California Institute of Technology, Pasadena, California 91125
}

\begin{abstract}
This paper studies the relationship between height, volume and stress distribution in a superpressure pumpkin balloon with the differential pressure applied to the balloon. Two different approaches are presented. A simple two-dimensional solution based on previous work and a novel, detailed finite element simulation that provides three-dimensional solutions are investigated. It is found that the finite element solution provides a much better agreement with experimental results for the case of a flat facet ULDB balloon. It is also found that a region of tensile hoop stress remains in the crown region of the balloon until the bottom pressure becomes negative. This region prevents the formation of clefts in the balloon and keeps its shape axisymmetric.
\end{abstract}

\section{Introduction}

Large balloons have an important role in atmospheric research, astrophysics, astronomy, etc. NASA's development of a large payload, high altitude and long duration balloon, the so called Ultra Long Duration Balloon, or ULDB, ${ }^{1}$ centers on a pumpkin shaped superpressure design. The idea behind the pumpkin balloon, first proposed by J. Nott ${ }^{2}$ is to use a light film as a gas barrier together with reinforced tendons to which much of the film stress is transferred. However, several pumpkin balloons have been unable to take up the desired, cyclically symmetrical equilibrium configuration; instead, they settle into a distorted anomalous shape. Thus, the successful design of pumpkin balloons requires a thorough understanding of multiple stable equilibria and the prediction of the evolution of the balloon shape.

The aim of this research is to study how the geometry (shape, height, volume) and mechanical parameters (strain and stress distribution) of a pumpkin balloon vary as the balloon rises in the atmosphere. Two different simulation methods are investigated in this paper: a finite-difference solution of the differential equation for an axi-symmetric balloon that carries stress purely in the meridional direction and a fully threedimensional finite-element solution that incorporates wrinkling and frictionless contact. In both cases the loading on the balloon is a non-uniform differential pressure with constant gradient along the height. Comparisons between these two solutions and experimentally observed shapes, from the test shown in Figure 1, will be made.

The paper is organized as follows. Section II presents a brief review of the literature on the shape of partially inflated balloons. Section III describes the test balloon. Section IV presents the axisymmetric formulation and solution scheme. Section V introduces the finite-element solution and presents an extensive set of results for the test balloon, that provide detailed insights into the behavior of partially inflated balloons. Section VI concludes the paper.

\section{Background}

Pioneering studies of the structural mechanics of balloons were carried out by Smalley, ${ }^{3}$ who proposed a direct equilibrium approach (similar to that adopted in Section IV), and Nishimura, Ohai and Ogita, ${ }^{4}$ who proposed a variational formulation.

\footnotetext{
${ }^{*}$ Research Student, Graduate Aeronautical Laboratories, 1200 East California Boulevard.

${ }^{\dagger}$ Professor of Aeronautics and Civil Engineering, Graduate Aeronautical Laboratories, 1200 East California Boulevard, Mail
} Code 301-46. e-mail sergiop@caltech.edu. Fellow of AIAA. 


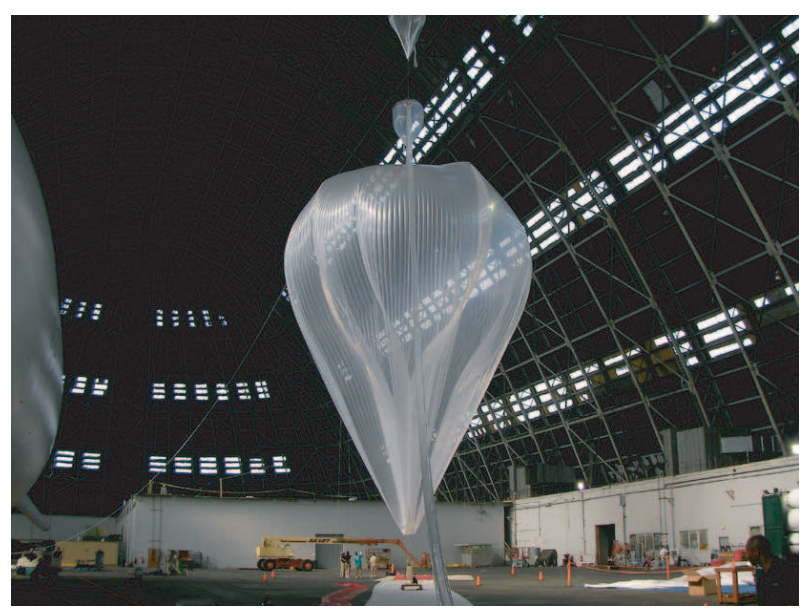

Figure 1. Partially-inflated shape of $27 \mathrm{~m}$ diameter balloon (courtesy of NASA BPO).

The equilibrium equations for an axisymmetric balloon with so-called natural shape (i.e. a balloon that carries the pressure load purely in the meridional direction) can be cast in terms of a first-order nonlinear differential equation. Two numerical solutions to this equation were discussed by Rand. ${ }^{5}$ The first is a "shooting" method solution, where certain variables are assumed at the start of the integration and a check of the final radius and angle is made at the end. If the check is not satisfied, the assumed starting values are changed and the process repeated. The difficulty of the technique is that the equations may become numerically unstable in the "rope" section at the bottom of the balloon. The alternative approach starts from the observation that, regardless of altitude, the apex of the balloon is always fully inflated. Hence the integration starts with an assumed pressure at the apex. As the integration proceeds down the balloon, the circumferential load required to satisfy the equilibrium equations is monitored. This force will become negative when excess material is available. At this point the force is set to zero and the calculation proceeds to generate the "natural shape" with no circumferential stress. Due to the delicate balance between the applied forces and the extent of the integration, the numerical procedure selected was a modified RungeKutta technique. Figure 2 shows a sequence of results obtained by Rand. Archimedes' principle states that for equilibrium, the balloon must displace an amount of air equal to its weight, hence $W_{t o t}=L i f t=b V$ where $V$ is the volume of the balloon, $W_{\text {tot }}$ is the total weight, and $b=g\left(\rho_{\text {out }}-\rho_{\text {in }}\right)$ is the specific buoyancy of the lifting gas. The subscript $d$ indicates a quantity related to the design shape. At float $b=b_{d}$ and $V=V_{d}$. When $V<V_{d}$, equilibrium requires that $b V=b_{d} V_{d}$. Thus $b$ is adjusted so that $b=b_{d} V_{d} / V$ and hence this parameter describes the ascent shape.

Baginski $^{6-8}$ solved the problem of determining the equilibrium shape of a strained balloon using the principle of minimum total potential energy. The total potential energy $\varepsilon$ of a strained, inflated balloon in a configuration $\varphi$ is given by

$$
\varepsilon(\varphi)=\varepsilon_{P}+\varepsilon_{f}+\varepsilon_{t}+\varepsilon_{t o p}+S_{t}^{*}+S_{f}^{*}
$$

where $\varepsilon_{P}$ is the hydrostatic pressure potential of the lifting gas, $\varepsilon_{f}$ the gravitational potential energy of the film, $\varepsilon_{t}$ the gravitational potential energy of the load tendons, $\varepsilon_{t o p}$ the gravitational potential energy of the apex fitting, $S_{t}^{*}$ the relaxed strain energy of the tendons, and $S_{f}^{*}$ the relaxed strain energy of film. Then, the following problem is defined

$$
\min \varepsilon(\varphi) \quad \text { where } \varphi \in C_{s}
$$

where $C_{s}$ denotes the class of feasible balloon shapes. Any boundary conditions or symmetry conditions that are to be imposed on the particular shapes of interest can be built into $C_{s} . S\left(V, n, r_{g}\right)$ denotes a balloon with volume $V, n$ lobes and $r_{g}$ gores per lobe. The total number of gores, $n_{g}$, can be expressed as $n_{g}=r_{g} n$. As an example of the shapes computed by Baginski, a partially inflated balloon with periodic lobes $S\left(0.4 V_{d}, 9,3\right)$ is shown in Figure 3. 


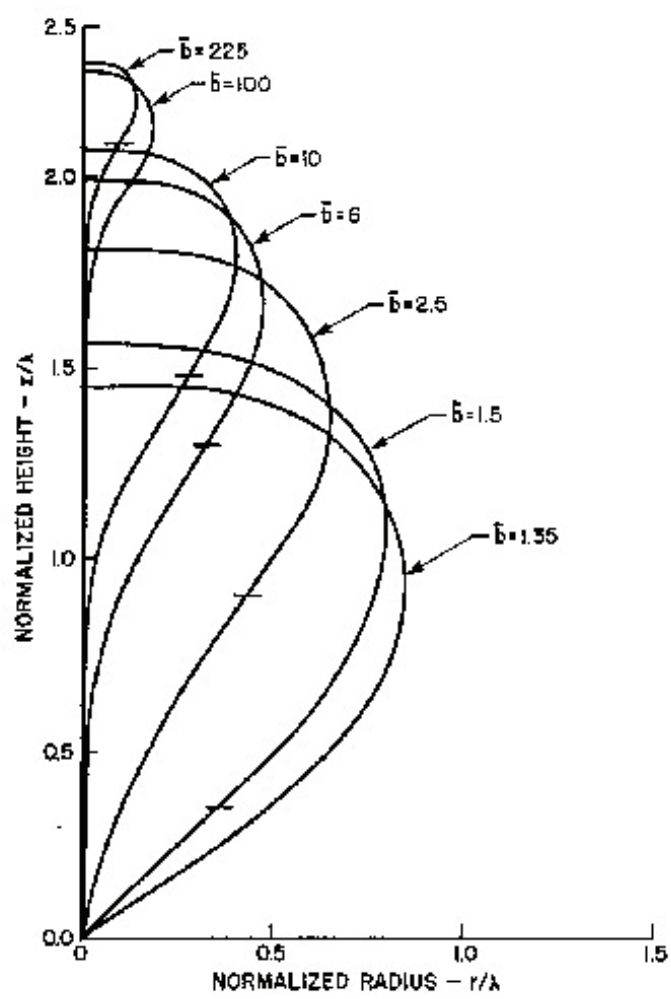

Figure 2. Natural shape balloons, $\bar{b}=V_{d} / V .^{5}$

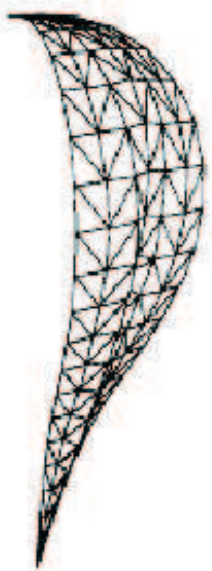

(a)

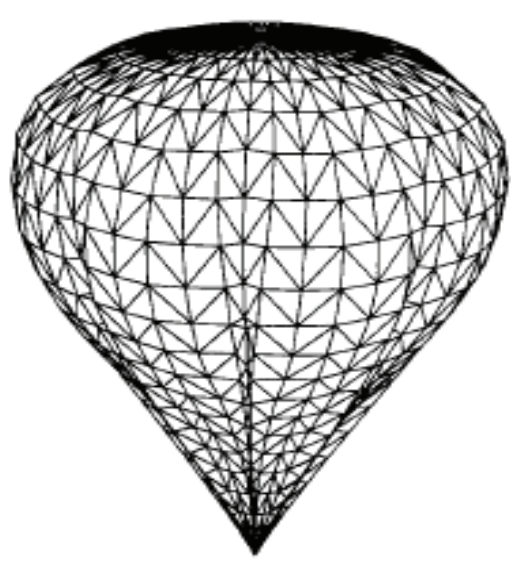

(b)

Figure 3. 27-gore pumpkin balloon $S\left(0.4 V_{d}, 9,3\right)$ : (a) 3-gore lobe from $S$ (b) Balloon configuration $S .^{\mathbf{8}}$ 


\section{Test Balloon}

A "flat lobe" ULDB balloon with $S_{0}=35.93 \mathrm{~m}$ and consisting of 200 identical lobes was recently tested indoors by the ULDB team. The balloon was initially filled with $18.3 \mathrm{~kg}$ of helium at atmospheric pressure and then fully inflated by adding air. The fully inflated volume was approximately $7000 \mathrm{~m}^{3}$ and the corresponding differential density between the mixed helium and air inside the balloon and the air outside was $-0.162 \mathrm{~kg} / \mathrm{m}^{3}$. The gas inside the balloon was slowly pumped out from an orifice in the bottom plate and the shape of the balloon was observed during the ensuing deflation process.

A simple description of this test balloon can be obtained by placing the tendons on the surface of an isotensoid (i.e. the equilibrium shape of a balloon that carries uniform pressure only through meridional stress $^{9-11}$ ) and by designing the cutting pattern such that the lobes span across the tendons. The following relationships ${ }^{12}$ exist between the equatorial radius of the isotensoid, $r_{\text {equ }}$, and its height, $z_{h}$, volume, $V$, and meridional arc length, $S_{0}$ :

$$
\begin{array}{r}
z_{h}=2 \times 0.5991 \times r_{e q u} \\
V=2.7458 \times r_{e q u}^{3} \\
S_{0}=2.6221 \times r_{e q u}
\end{array}
$$

The values of these parameters in the case of the test balloon are presented in Table 1. The cutting pattern for the lobes is thus obtained by noting that the subtended angle between adjacent meridians is $\theta=2 \pi / n=\frac{360^{\circ}}{200}=1.8^{\circ}$. The width of the flat cutting pattern is obtained by considering the distance between corresponding points on the two meridians. For example, we have $z_{A}=r_{e q u} \sin \frac{\theta}{2}$. Finally the lobe is copied and rotated $n-1$ times to form a periodically symmetric surface, see Figure 4 . The test balloon was made of linear low density polyethylene (LLDPE) for the film and braided PBO for the tendons. The material properties are given in Table 2.
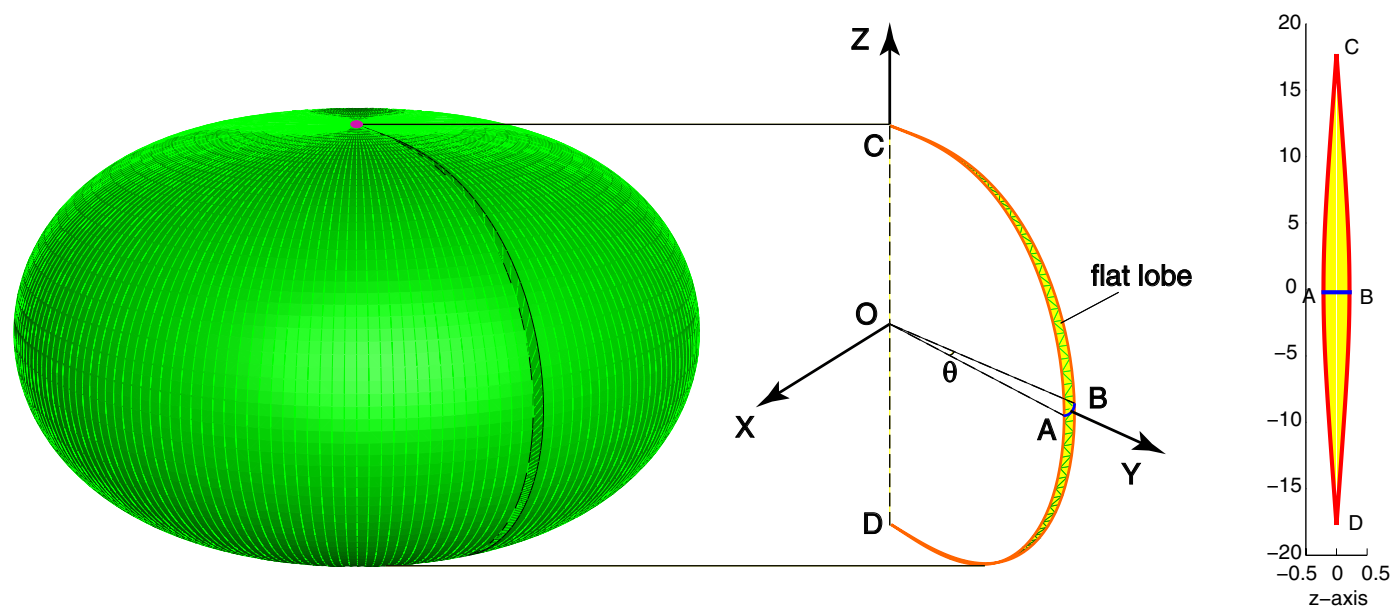

Figure 4. Definition of lobe cutting pattern

Table 1. Geometry of test balloon

\begin{tabular}{lc}
\hline \hline Diameter $(\mathrm{m}), 2 \times r_{\text {equ }}$ & 27.41 \\
Height $(\mathrm{m}), z_{h}$ & 16.42 \\
Gore Length $(\mathrm{m}), S_{0}$ & 35.93 \\
Volume $\left(\mathrm{m}^{3}\right), V$ & 7060 \\
Radius of apex plate $(\mathrm{m})$ & 0.406 \\
\hline \hline
\end{tabular}


Table 2. Material properties for test balloon

\begin{tabular}{lc}
\hline \hline \multicolumn{2}{c}{ LLDPE film } \\
\hline Thickness of film, $(\mathrm{mm})$ & 0.013 \\
Young's Modulus, $\left(\mathrm{N} / \mathrm{mm}^{2}\right)$ & 138.7 \\
Poisson's ratio, $\nu$ & 0.56 \\
Density, $\rho\left(\mathrm{kg} / \mathrm{m}^{3}\right)$ & 920 \\
\hline \multicolumn{1}{c}{$\mathrm{PBO}$ tendons } \\
\hline Area, $\left(\mathrm{mm}^{2}\right) \quad 40$ \\
Young's Modulus, $\left(\mathrm{N} / \mathrm{mm}^{2}\right)$ & 4250 \\
Density, $\rho\left(\mathrm{kg} / \mathrm{m}^{3}\right)$ & 300 \\
\hline \hline
\end{tabular}

\section{Axisymmetric Solution}

This section briefly reviews the analytical formulation for an axisymmetric balloon with zero hoop stress. Its surface is defined by the meridional profile, $r(z)$, Figure 5 . The radius of principal curvature in the meridional direction is $r_{1}$ and the second radius of principal curvature is $r_{2} ; s$ represents the arc length in the meridional direction, measured from the apex. The angle $\phi$ measures the colatitude, while the angle $\theta$ measures the longitude. From Figure 5(a)

$$
r=r_{2} \sin \phi
$$

and the meridional curvature has the standard expression

$$
\frac{1}{r_{1}}=-\frac{d^{2} r / d z^{2}}{\left[1+(d r / d z)^{2}\right]^{\frac{3}{2}}}
$$

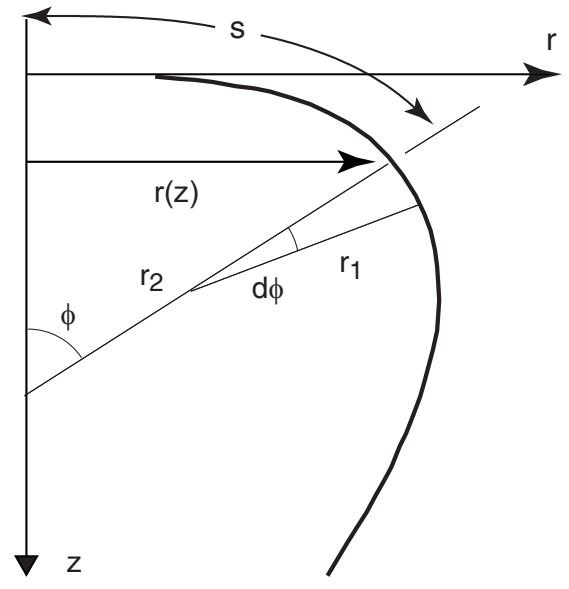

(a)

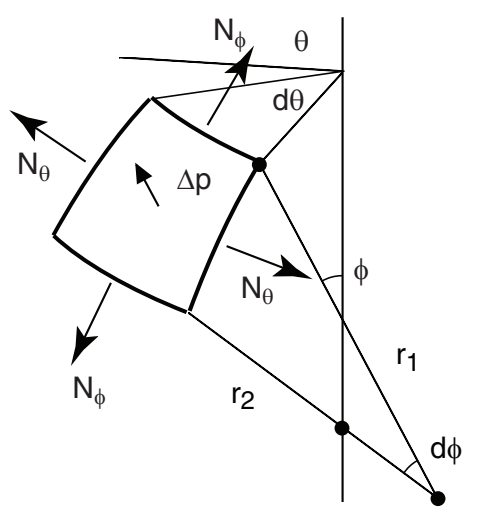

(b)

Figure 5. (a) Geometry of axisymmetric balloon; (b) equilibrium of infinitesimal element.

The meridional and hoop tensions per unit length are denoted by $N_{\phi}$ and $N_{\theta}$ respectively, the normal pressure is denoted by $\Delta p$, and there is no shear for symmetry, see Figure $5(\mathrm{~b})$. In general, they are related by the three equations of equilibrium ${ }^{13}$

$$
\frac{\partial\left(r_{2} \sin \phi N_{\phi}\right)}{\partial \phi}-r_{1} \cos \phi N_{\theta}=0
$$


in the meridional direction,

$$
r_{1} \frac{\partial N_{\theta}}{\partial \theta}=0
$$

in the hoop direction, and

$$
\frac{N_{\phi}}{r_{1}}+\frac{N_{\theta}}{r_{2}}=\Delta p
$$

along the normal.

Since it has been assumed that the film has no hoop tension

$$
N_{\theta}=0
$$

Hence, Equation 9 is satisfied and Equation 10 becomes

$$
N_{\phi}=\Delta p r_{1}
$$

Substituting Equation 6, Equation 11 and Equation 12 into Equation 8 we get

$$
\frac{\partial\left(r N_{\phi}\right)}{\partial \phi}=0
$$

and solving this partial differential equation gives

$$
N_{\phi}=C(\theta) / r
$$

For symmetry, $N_{\phi}$ has to be independent of $\theta$ and hence

$$
N_{\phi}=C / r
$$

where $C$ is called the stress constant. It can be seen that there is a singular point at the crown of the balloon, where the above equation gives an infinite $N_{\phi}$.

Substituting Equation 12 into Equation 15, and then Equation 7 for $r_{1}$ and rearranging gives the differential equation governing the profile of the axisymmetric balloon with zero hoop stress

$$
\frac{d^{2} r}{d z^{2}}+\frac{\Delta p}{C} r\left[1+(d r / d z)^{2}\right]^{\frac{3}{2}}=0
$$

This is an initial value problem because we know in advance only the boundary conditions at the crown, which can be expressed as

$$
r(0)=0, \quad\left(\frac{d r}{d z}\right)_{0}=\infty
$$

\section{A. Computation of Meridional Profile}

The total meridional arc length $S_{0}$ is assumed to be given and to remain constant. Hence throughout the analysis the envelope arc length $S_{\text {env }}$ should also be equal to $S_{0}$, Figure 6 . We postulate that the internal gas density remains constant when the pressure at the bottom, $\Delta p_{0}$ is decreased (this assumption would not be acceptable in the general case of a helium-filled balloon rising in the atmosphere but is acceptable to model the indoor experiments that have been carried out by the ULDB team). The differential pressure is then given by

$$
\Delta p=-\Delta \rho g\left(z-z_{h}\right)+\Delta p_{0}
$$

where $z_{h}$ is the height of the balloon and $\Delta \rho=\rho_{\text {out }}-\rho_{\text {in }}$. Since the boundary conditions are known only at the top, the integration of the differential equation should go from the top to the bottom.

Given the bottom differential pressure, $\Delta p_{0}$, the integration algorithm is as follows:

1 Guess the height of the balloon $z_{h}^{*}$ and then find the distribution of pressure difference $\Delta p$ from Equation 18;

2 Guess the stress constant $C^{*}$; 


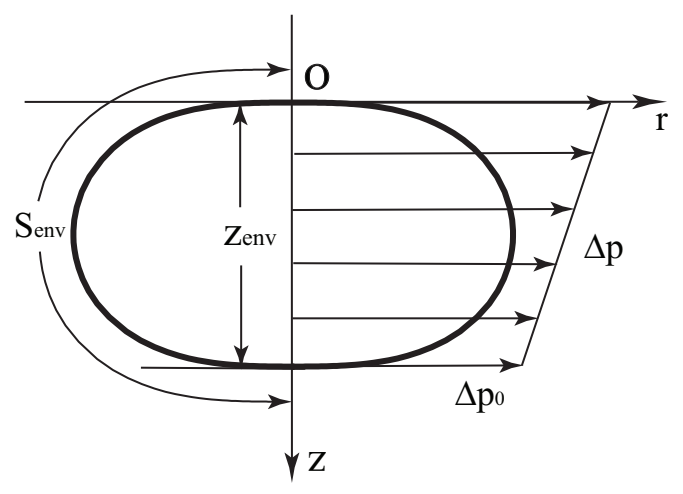

(a)

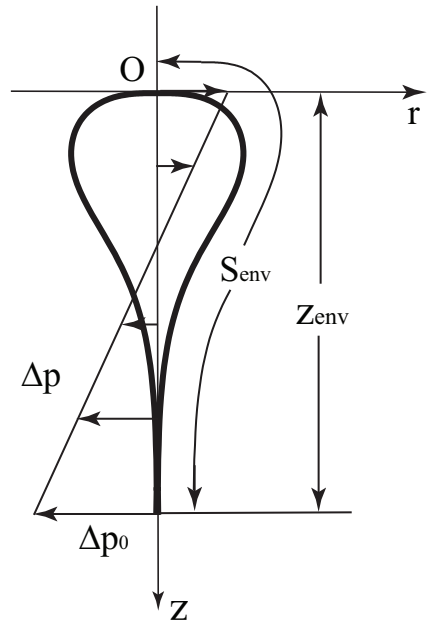

(b)

Figure 6. Assumed differential pressure distributions (a) fully inflated; (b) partially inflated.

3 Find trial envelope heights $z_{e n v}$ and envelope arc length $S_{e n v}$ by solving Equation 16 by finite differences;

4 If $S_{\text {env }}=S_{0}$, then the solution continues to the next step; otherwise it returns to step 2 ;

5 If $z_{e n v}=z_{h}^{*}$, then the solution is completed; otherwise it returns to step 1 .

If the balloon is fully inflated and pressurized, the bottom differential pressure $\Delta p_{0}$ is positive and the difference between top and bottom pressures is small. Hence the zero pressure point is at $z \gg z_{\mathrm{env}}$, see Figure 6(a). The process of deflation reaches a critical point when the bottom differential pressure turns negative and the difference between top and bottom pressures becomes significant. From this point on, the zero pressure point lies within the envelope of the balloon and the bottom part of the balloon is subjected to negative pressure, see Figure 6(b).

\section{B. Predictions for Test Balloon}

We have used the above algorithm to compute the key geometric parameters of the test balloon at different differential pressures. The results are presented in Table 3, and a selection of the corresponding shapes is shown in Figure 7. Note that the top set of results corresponds to the isotensoid shape, as the pressure gradient has been set to zero for this particular analysis.

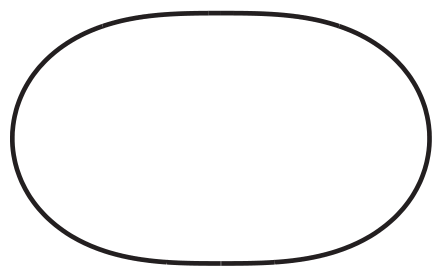

(a)

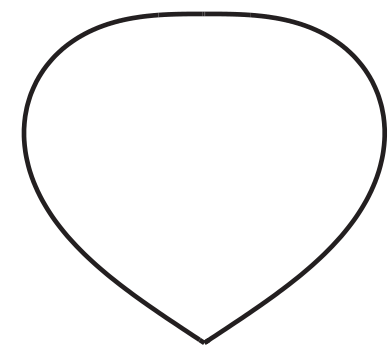

(b)

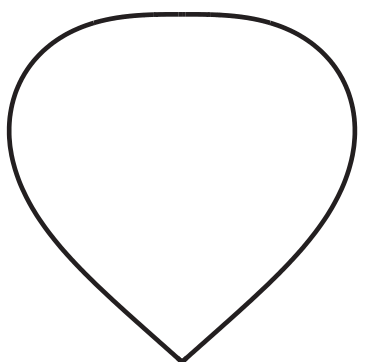

(c)

Figure 7. Shapes of axisymmetric balloons with zero hoop stress: (a) no. 1; (b) no. 2; (c) no. 8 (the numbering corresponds to the rows in Table 3) 
Table 3. Axisymmetric predictions for test balloon

\begin{tabular}{|c|c|c|c|c|c|}
\hline \hline No. & $\begin{array}{c}\text { Bottom pressure } \\
\Delta p_{0}(\mathrm{~Pa})\end{array}$ & $\begin{array}{c}\text { Pressure gradient } \\
\Delta \rho(\mathrm{Pa} / \mathrm{m})\end{array}$ & $\begin{array}{c}\text { Volume } \\
V\left(\mathrm{~m}^{3}\right)\end{array}$ & $\begin{array}{c}\text { Max radius } \\
r_{\max }(\mathrm{m})\end{array}$ & $\begin{array}{l}\text { Height } \\
z_{h}(\mathrm{~m})\end{array}$ \\
\hline 1 & 10 & 0 & 7044.9 & 13.686 & 16.424 \\
\hline 2 & 7 & 0.162 & 6217.03 & 12.157 & 22.131 \\
\hline 3 & 4 & 0.162 & 6114.40 & 12.024 & 22.588 \\
\hline 4 & 1 & 0.162 & 5959.07 & 11.843 & 23.026 \\
\hline 5 & 0 & 0.162 & 5931.90 & 11.800 & 23.197 \\
\hline 6 & -0.5 & 0.162 & 5885.98 & 11.755 & 23.264 \\
\hline 7 & -1 & 0.162 & 5851.23 & 11.724 & 23.370 \\
\hline 8 & -1.5 & 0.162 & 5789.70 & 11.668 & 23.440 \\
\hline 9 & -2 & 0.162 & 5725.924 & 11.609 & 23.485 \\
\hline 10 & -2.5 & 0.162 & 5662.100 & 11.553 & 23.553 \\
\hline
\end{tabular}

\section{ABAQUS/Explicit Simulations}

We have used ABAQUS/Explicit (version 6.7) to simulate the behaviour of the test balloon as it is deflated. In general, ABAQUS/Explicit will compute the dynamic response of a structure subject to timevarying loads or boundary conditions. However, by ensuring that the loading rate is sufficiently slow, the dynamic response of the structure is sufficiently small that the response becomes effectively quasi-static. The results presented in this section were obtained by carrying out the quickest possible quasi-static simulations while ensuring that the kinetic energy is only a small fraction of the strain energy.

\section{A. Simulation Details}

The test balloon was modelled using the reduced integration three-dimensional, four-node elements M3D4R for the balloon film and three-dimensional, two-node pin-jointed bars T3D2 for the tendons. Each tendon was divided into 70 elements and each lobe was modeled with a mesh of 140 elements. Hence the complete model consisted of 28,000 elements and approximately 14,000 nodes.

The three translational degrees of freedom of the node at the bottom of the balloon were fixed. The two in-plane translational degrees of freedom of the node at the crown of balloon were also fixed. All other degrees of freedom were left unrestrained.

To define the lobe geometry, the initial geometry of the lobe was mapped onto the flat cutting pattern of the lobe (in the reference coordinate system) using the command: "Initial conditions, Type=Ref Coordinate". This command sets up a one-to-one correspondence between elements in the two coordinate systems, see Figure 8. The initial stresses and strains in the initial configuration are computed automatically.

A user-defined subroutine (Vumat) was used to model wrinkling in the film and thus avoid compressive stresses. A mixed wrinkling criterion based on the variable Poisson's ratio method ${ }^{14,15}$ was adopted. Denoting the constitutive model for the membrane by $\{\sigma\}=[D]\{\varepsilon\}$, and the major and minor principal stresses by $\sigma_{1}, \sigma_{2}$, this subroutine recursively modifies the matrix $D$ according to the state (wrinkled, taut or slack) of each element, as follows.

- $\sigma_{2}>0$ : the membrane is taut, hence

$$
D_{t}=\frac{E}{1-\nu^{2}}\left[\begin{array}{ccc}
1 & \nu & 0 \\
\nu & 1 & 0 \\
0 & 0 & (1-\nu) / 2
\end{array}\right]
$$

- $\sigma_{2} \leq 0$ and $\varepsilon_{1}>0$ : the membrane is wrinkled, hence 


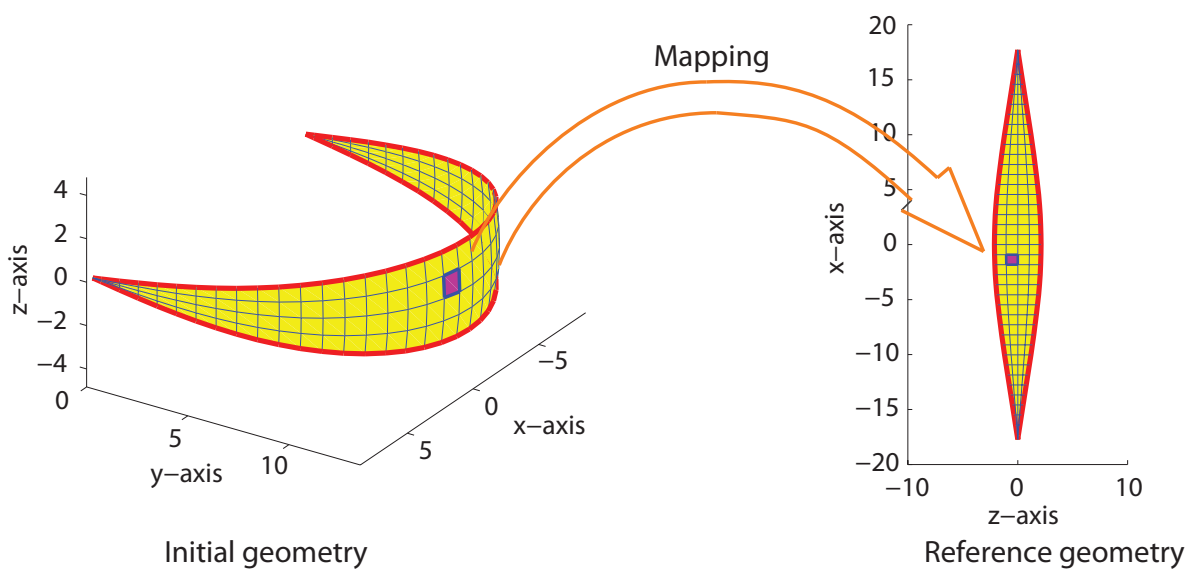

Figure 8. Initial geometry and reference geometry.

$$
D_{w}=\frac{E}{4}\left[\begin{array}{ccc}
2(1+P) & 0 & Q \\
0 & 2(1-P) & Q \\
Q & Q & 1
\end{array}\right]
$$

where $P=\left(\epsilon_{x}-\epsilon_{y}\right) /\left(\epsilon_{1}-\epsilon_{2}\right)$ and $Q=\gamma_{x y} /\left(\epsilon_{1}-\epsilon_{2}\right)$

- $\varepsilon_{1} \leq 0$ and $\sigma_{2}<0$ : the membrane is slack and hence $D_{s}=0$

It is also essential to model contact, to prevent the penetration of different parts of the membrane through other parts. General contact was defined for the surface of the balloon interacting with itself, using the option "Contact Inclusions".

During the simulation the rate of variation of any loads has to be sufficiently slow that the kinetic energy in the whole structure is negligibly small; also, the time-step increment has to be sufficiently small to avoid spurious effects due to stress waves. ABAQUS/Explicit will automatically calculate these increments, but linear bulk viscosity, defined by linear viscosity coefficient, is introduced to dampen any oscillations. This enhances stability but requires smaller time increments.

\section{B. Results}

Starting from a fully-inflated balloon with a uniform pressure of $10 \mathrm{~Pa}$, a constant pressure gradient of $0.162 \mathrm{~Pa} / \mathrm{m}$ was imposed and then the bottom differential pressure was decreased in steps of decreasing size down to $-2.5 \mathrm{~Pa}$. The results are presented in Table 4.

The linear viscosity coefficient should always be kept as small as possible to avoid affecting the equilibrium shape of the structure, however its value had to be increased when the bottom pressure became negative to avoid that the computation stops due to large distortion of the elements. The energy variation throughout the simulation is plotted in Figure 9. Note that the artificial energy, used to control hourglassing in the reduced integration membrane elements, decreases once the deflated shape of the balloon has developed. Also note that the amount of viscous energy that is required to keep the simulation going gradually increases as the balloon becomes more unstable with more and more clefts.

Figure 10 shows a series of snapshots of the deflated balloon, with details provided in Table 5, and Figures 11 and 12 show the corresponding distributions of meridional and hoop stress in the membrane. An interesting observation from these figures is that the balloon was still essentially axisymmetric at Step 5, i.e. when the bottom pressure had become zero. Also note that, although much of the balloon is wrinkled, as the hoop stress has vanished, there is still a region of hoop tension near the crown of the balloon. Loss of symmetry and the onset of clefting occur when the hoop stress in this upper region becomes zero, which happens at a bottom pressure of around $-1 \mathrm{~Pa}$. 
Table 4. ABAQUS/Explicit simulation steps

\begin{tabular}{|c|c|c|c|c|}
\hline \hline Step No. & $\begin{array}{c}\text { Bottom pressure } \\
\Delta p_{0}(\mathrm{~Pa})\end{array}$ & $\begin{array}{c}\text { Pressure gradient } \\
\Delta \rho(\mathrm{Pa} / \mathrm{m})\end{array}$ & $\begin{array}{c}\text { Loading time } \\
(\mathrm{s})\end{array}$ & Viscosity coefficient \\
\hline 1 & 10 & 0 & 20 & 0.05 \\
\hline 2 & 7 & 0.162 & 20 & 0.05 \\
\hline 3 & 4 & 0.162 & 20 & 0.03 \\
\hline 4 & 1 & 0.162 & 20 & 0.02 \\
\hline 5 & 0 & 0.162 & $10+5^{a}$ & 0.02 \\
\hline 6 & -0.5 & 0.162 & $15+5^{a}$ & 0.02 \\
\hline 7 & -1 & 0.162 & $15+5^{a}$ & 0.08 \\
\hline 8 & -1.5 & 0.162 & 10 & 0.12 \\
\hline 9 & -2.0 & 0.162 & 10 & 0.25 \\
\hline 10 & -2.5 & 0.162 & 10 & 0.80 \\
\hline \hline
\end{tabular}

\footnotetext{
${ }^{a}$ The additional 5 seconds are at constant pressure, to test the stability of the result.
}
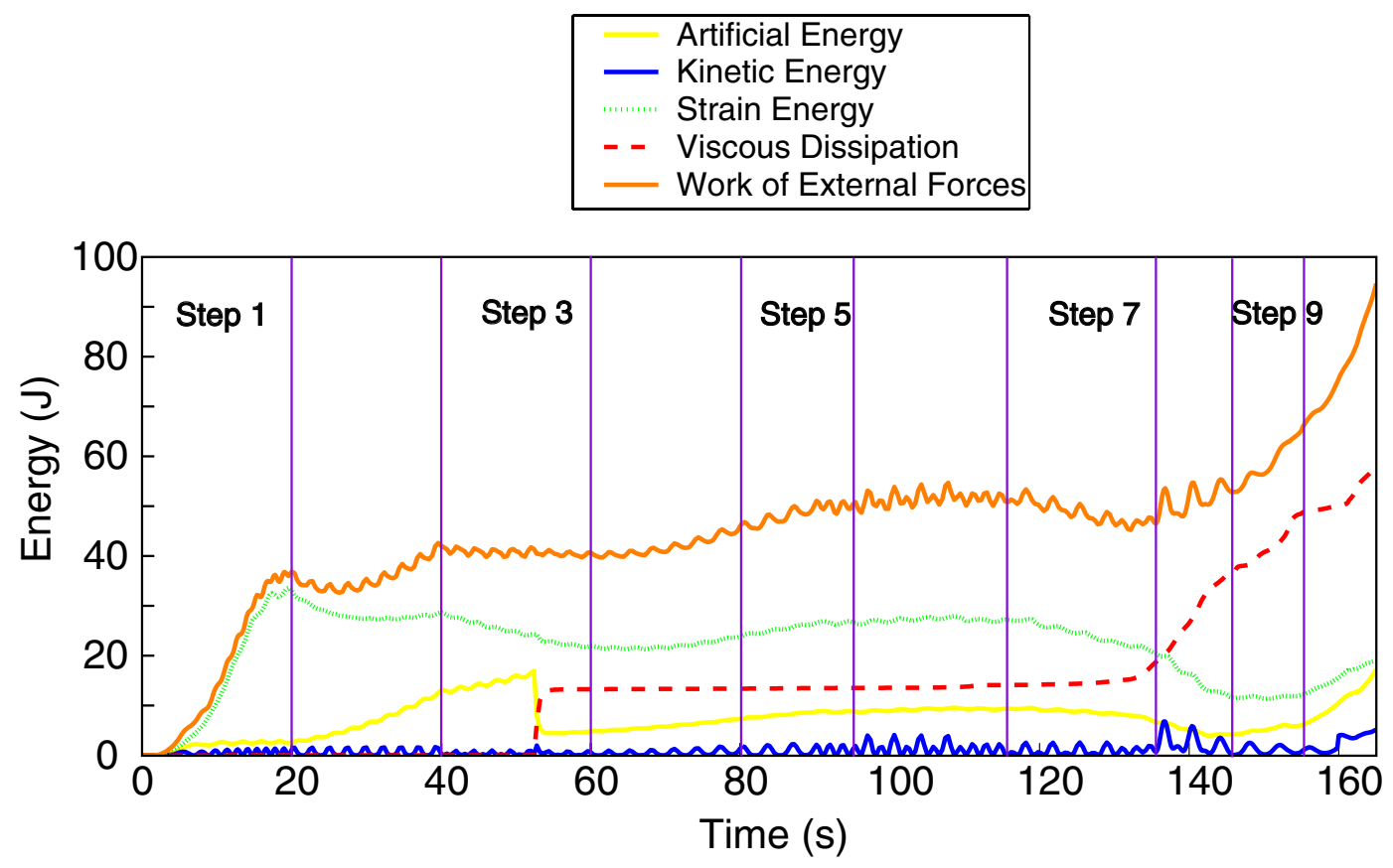

Figure 9. Energy variation during ABAQUS/Explicit simulation. 

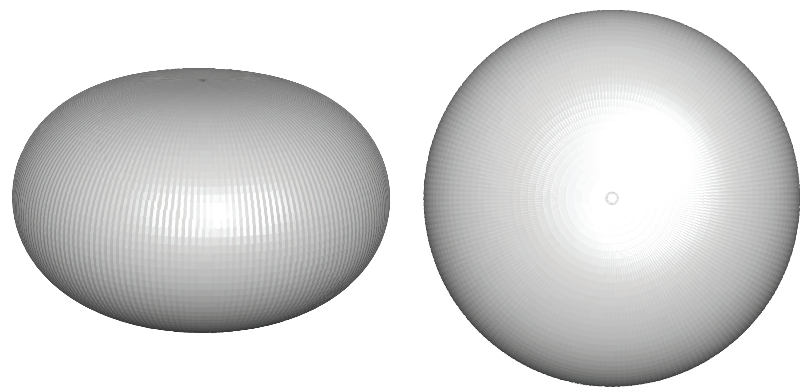

(a) Step 1

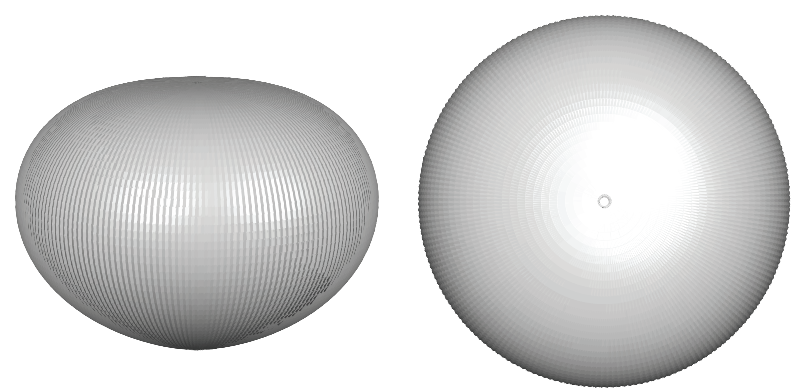

(b) Step 3
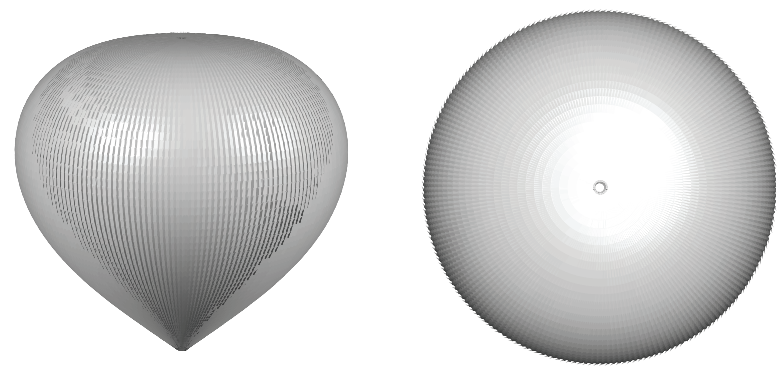

(c) Step 5
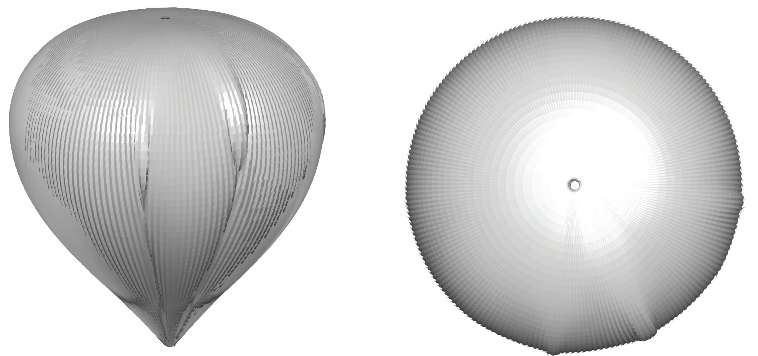

(e) Step 7

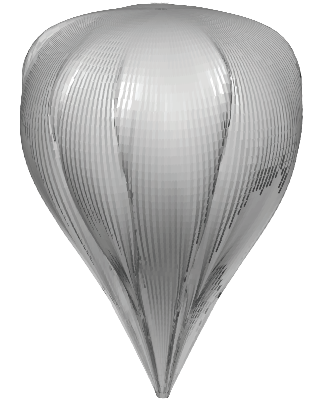

Side view

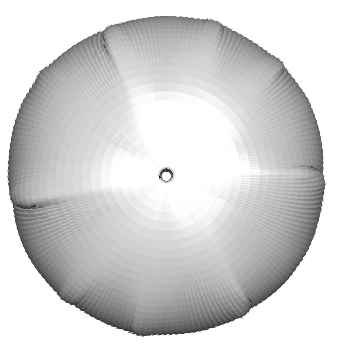

(f) Step $9 \quad$ Top view

Figure 10. Side and top views of test balloon, from simulation.

11 of 18 
Table 5. ABAQUS/Explicit predictions of key parameters of test balloon

\begin{tabular}{|c|c|c|c|c|}
\hline \hline Step No. & $\begin{array}{c}\text { Volume } \\
V\left(\mathrm{~m}^{3}\right)\end{array}$ & $\begin{array}{c}\text { Max radius to membrane } \\
(\mathrm{m})\end{array}$ & $\begin{array}{c}\text { Max radius to tendons } \\
(\mathrm{m})\end{array}$ & $\begin{array}{c}\text { Height } \\
z_{h}(\mathrm{~m})\end{array}$ \\
\hline 1 & 7078.5 & 13.611 & 13.581 & 16.763 \\
\hline 2 & 6966.2 & 13.214 & 13.156 & 18.845 \\
\hline 3 & 6841.7 & 12.983 & 12.837 & 19.729 \\
\hline 4 & 6379.7 & 12.344 & 12.256 & 21.718 \\
\hline 5 & 5877.1 & 11.753 & 11.656 & 23.160 \\
\hline 6 & 5507.1 & 11.372 & 11.266 & 23.981 \\
\hline 7 & 5048.0 & 10.940 & 10.860 & 24.832 \\
\hline 8 & 4279.7 & 10.163 & 10.100 & 26.111 \\
\hline 9 & 3436.2 & 9.346 & 9.313 & 27.139 \\
\hline 10 & 2387.5 & 8.374 & 8.421 & 28.364 \\
\hline \hline
\end{tabular}

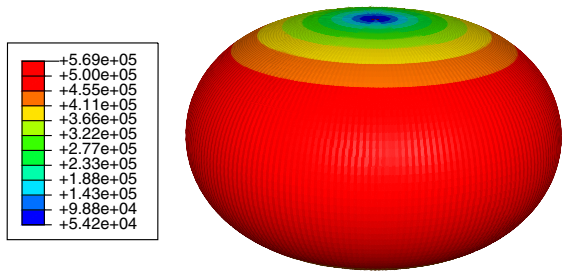

(a) Step 1
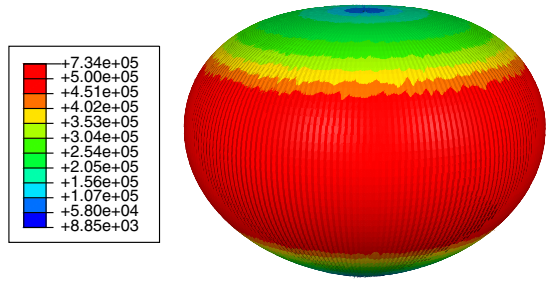

(b) Step 3

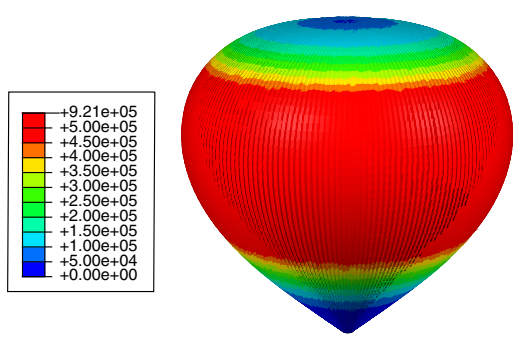

(c) Step 5

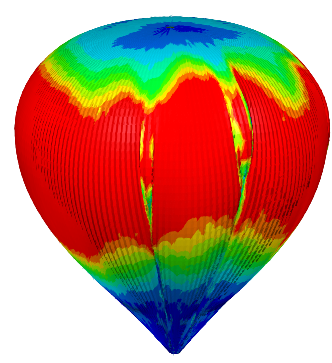

(d) Step 7

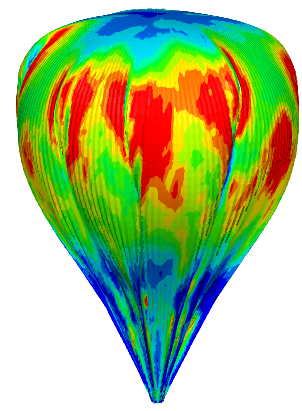

(e) Step 9

Figure 11. Distribution of meridional stress. 

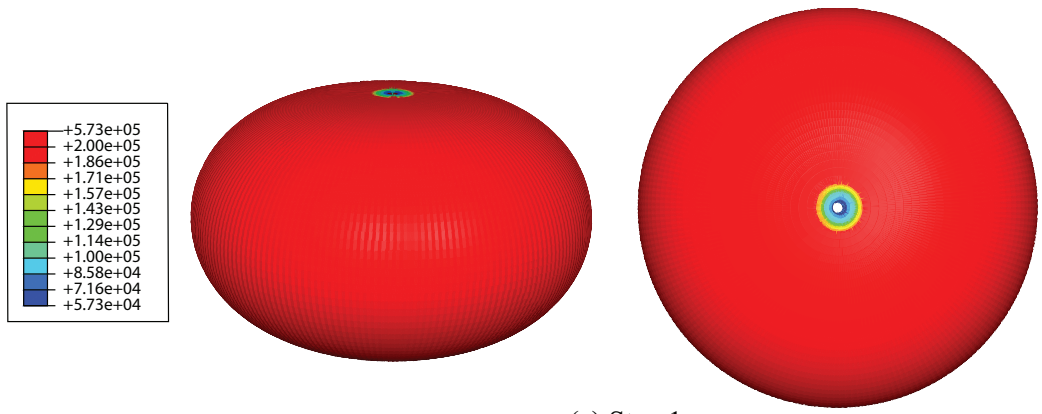

(a) Step 1
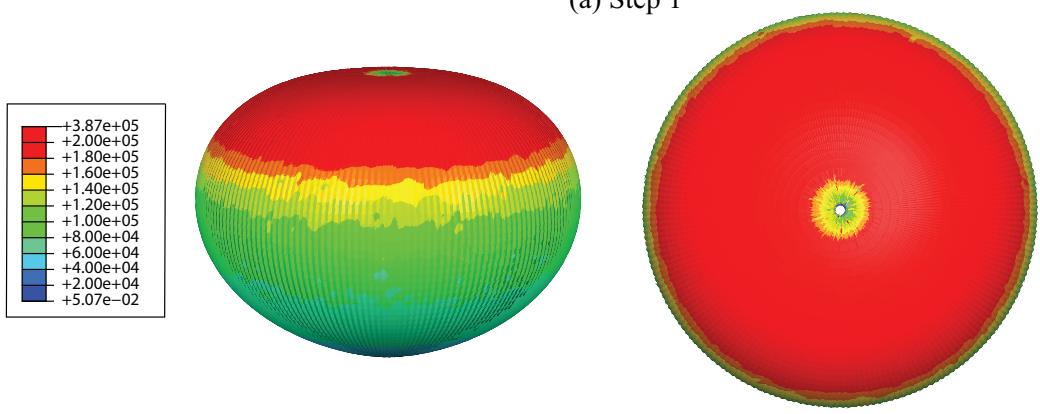

(b) Step 3
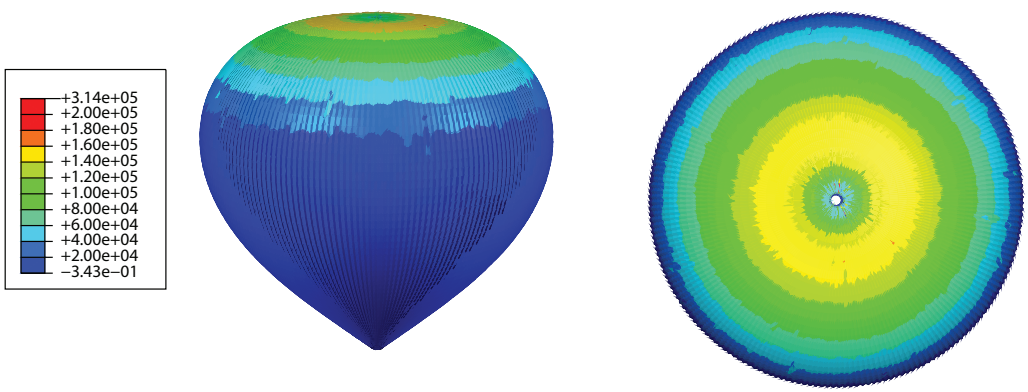

(c) Step 5
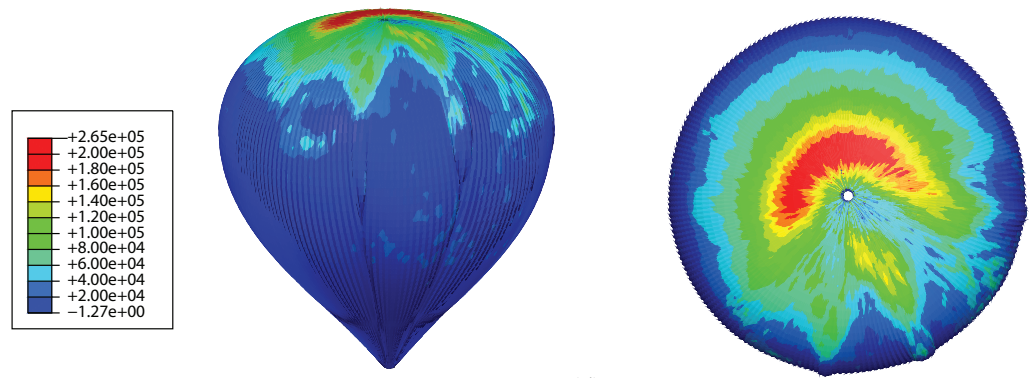

(d) Step 7
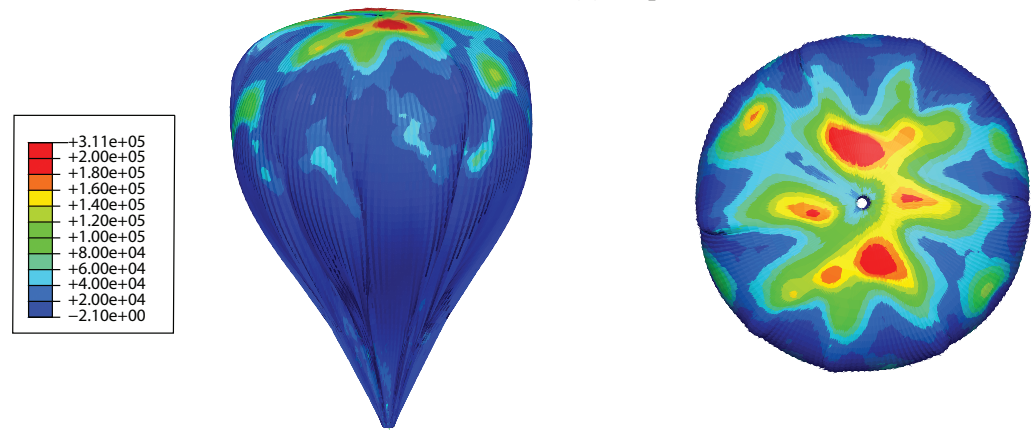

Side view

(e) Step 9

Top view

Figure 12. Distribution of hoop stress.

\section{3 of 18}

American Institute of Aeronautics and Astronautics 
Figures 13 and 14 show more details on some particular shapes that have been obtained from the simulation. We use a series of cross sections at different heights to show how the spare material in a heavily clefted balloon distributes itself in a non-symmetric way, forming inward pointing fins in regions of positive pressure. In Figure 14 it is noteworthy that outward pointing fins have formed in the bottom region, due to the negative pressure to which it is subjected.
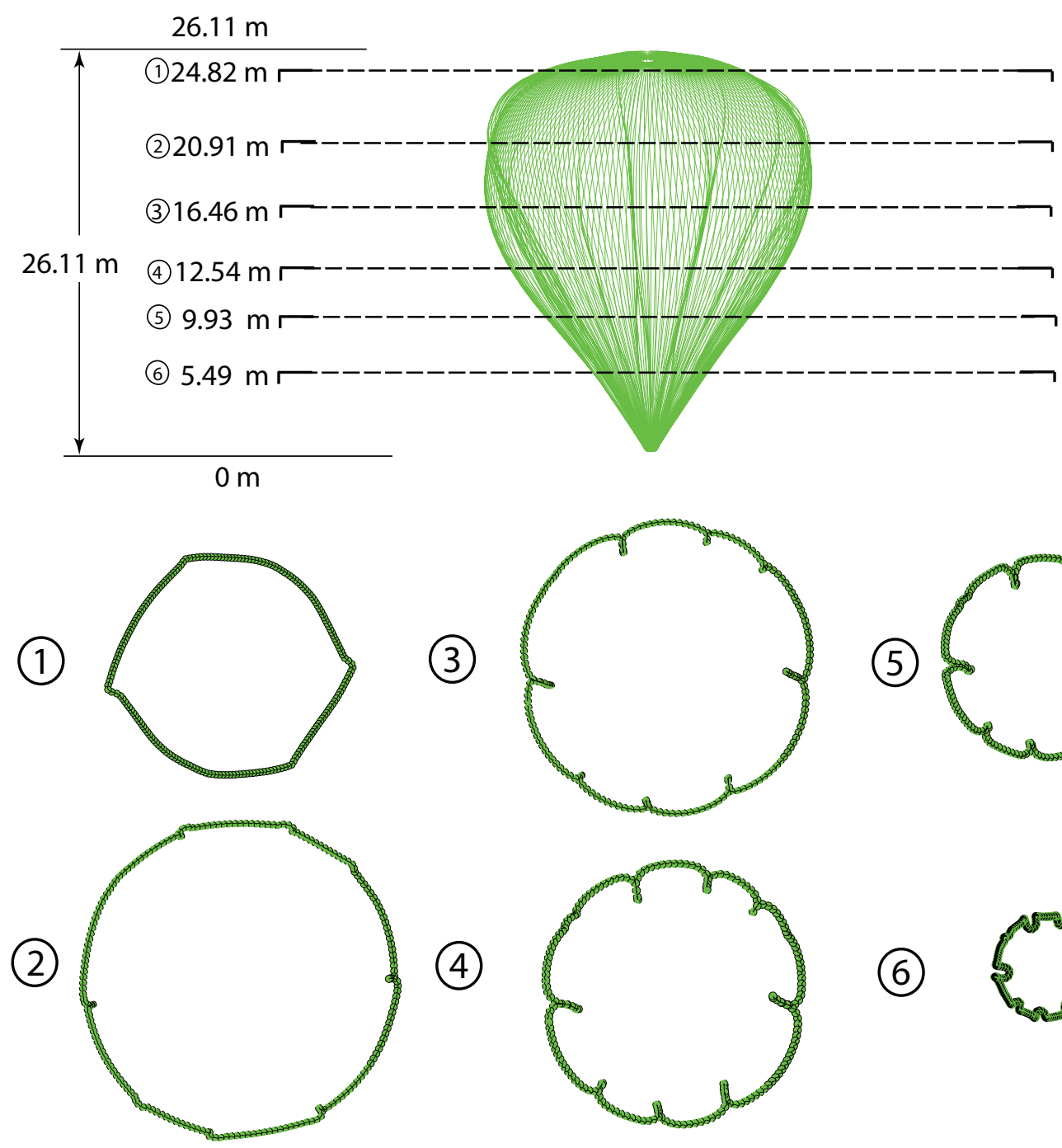

(3)

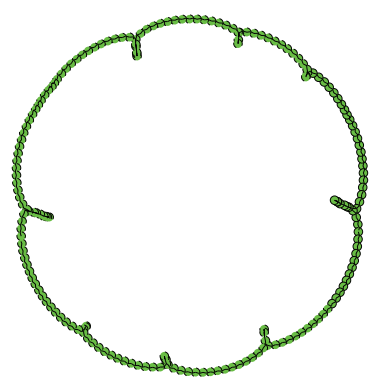

(4)

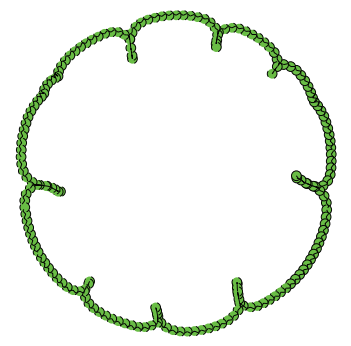

(5)

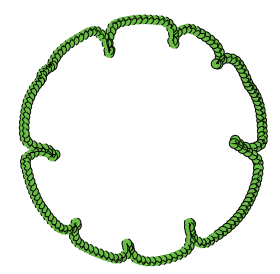

(6)

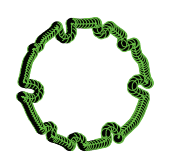

Figure 13. Shape and cross sections of balloon at Step 8

A visual comparison between these results and the test observations ${ }^{16}$ is shown in Figure 15. During deflation the test balloon showed clefts soon after the photo in Figure 15(a) was taken (at a bottom pressure of $1 \mathrm{~Pa}$ ) and here it is compared with the simulation results at the end of Step 4; all of the tendons lie in uniformly distributed planes. Figure 15(b) compares the simulation results at the end of Step 10, to the corresponding shape that was seen during the test.

\section{Discussion and Conclusion}

The objective of this study was to investigate the shape of partially inflated superpressure balloons. The relationships between height, volume, stress distribution and differential pressure distribution have allowed us to explore the formation of clefts in these balloons.

Two different approaches have been presented. A simple two-dimensional solution based on previous work and a novel finite element simulation that provides three-dimensional solutions. In the two-dimensional

\section{4 of 18}



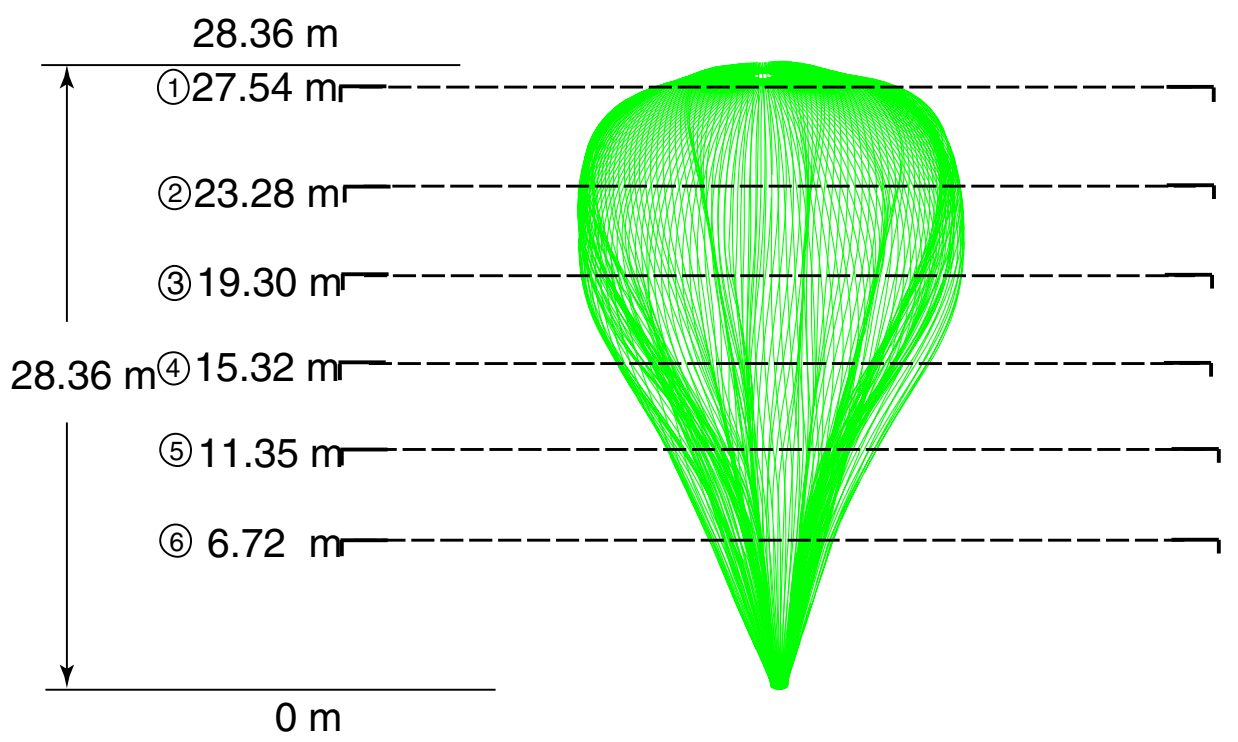

(1)
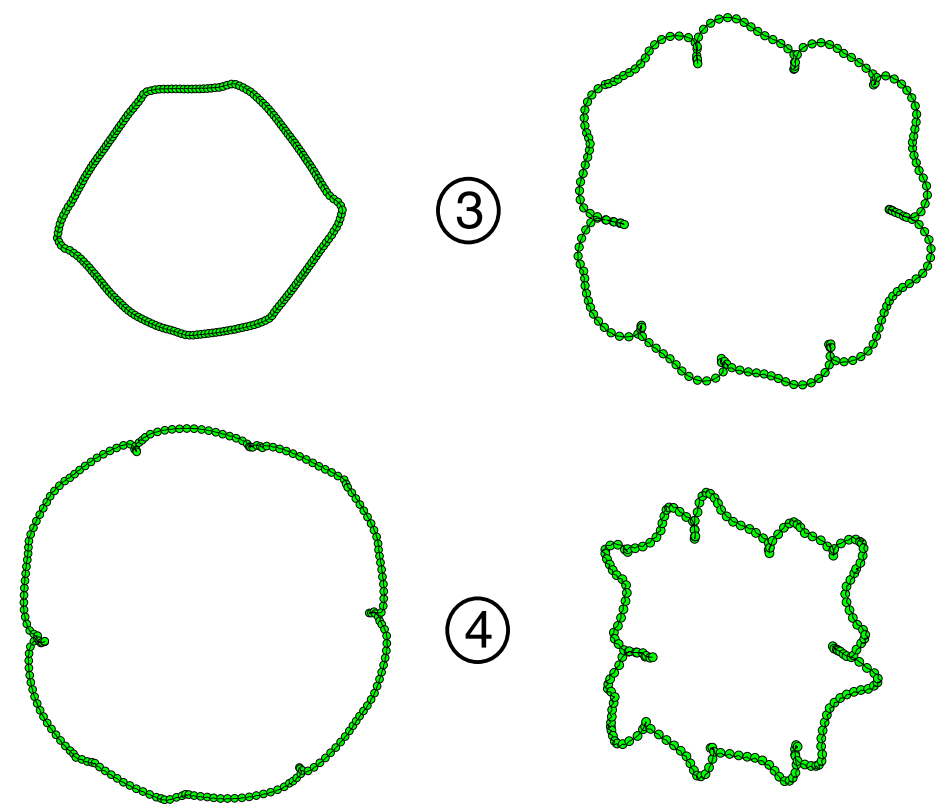

(3)

(4)

(5)

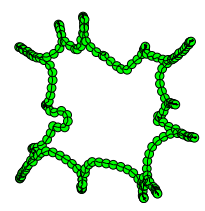

(6)

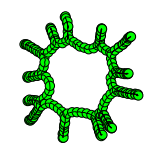

Figure 14. Shape and cross sections of balloon at Step 10. 

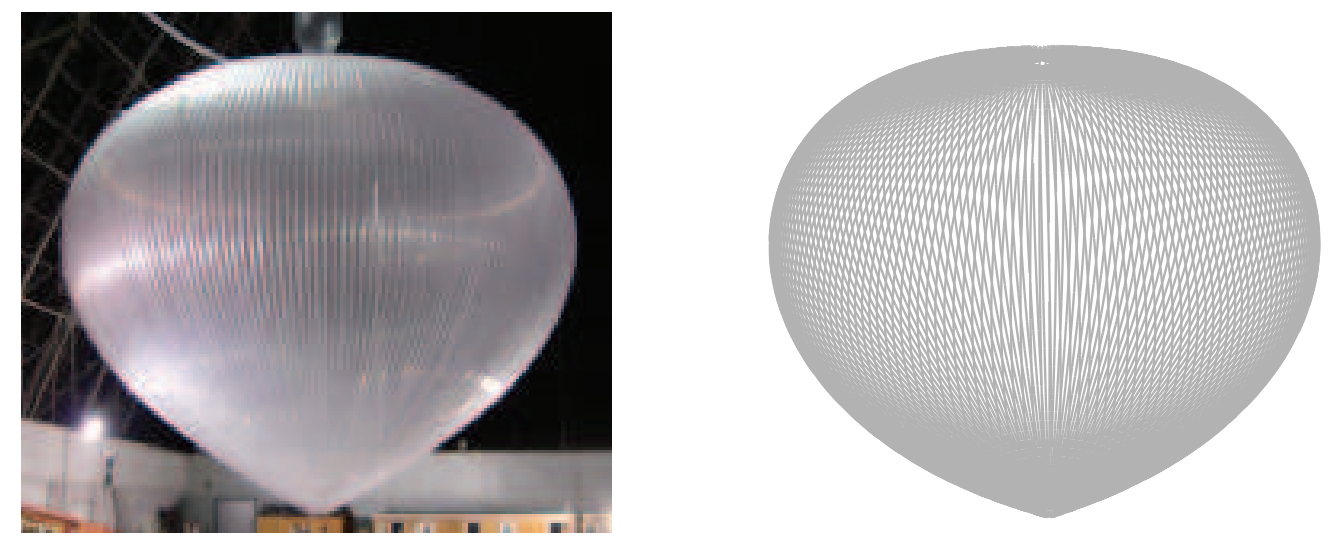

(a) Initial deployed shape
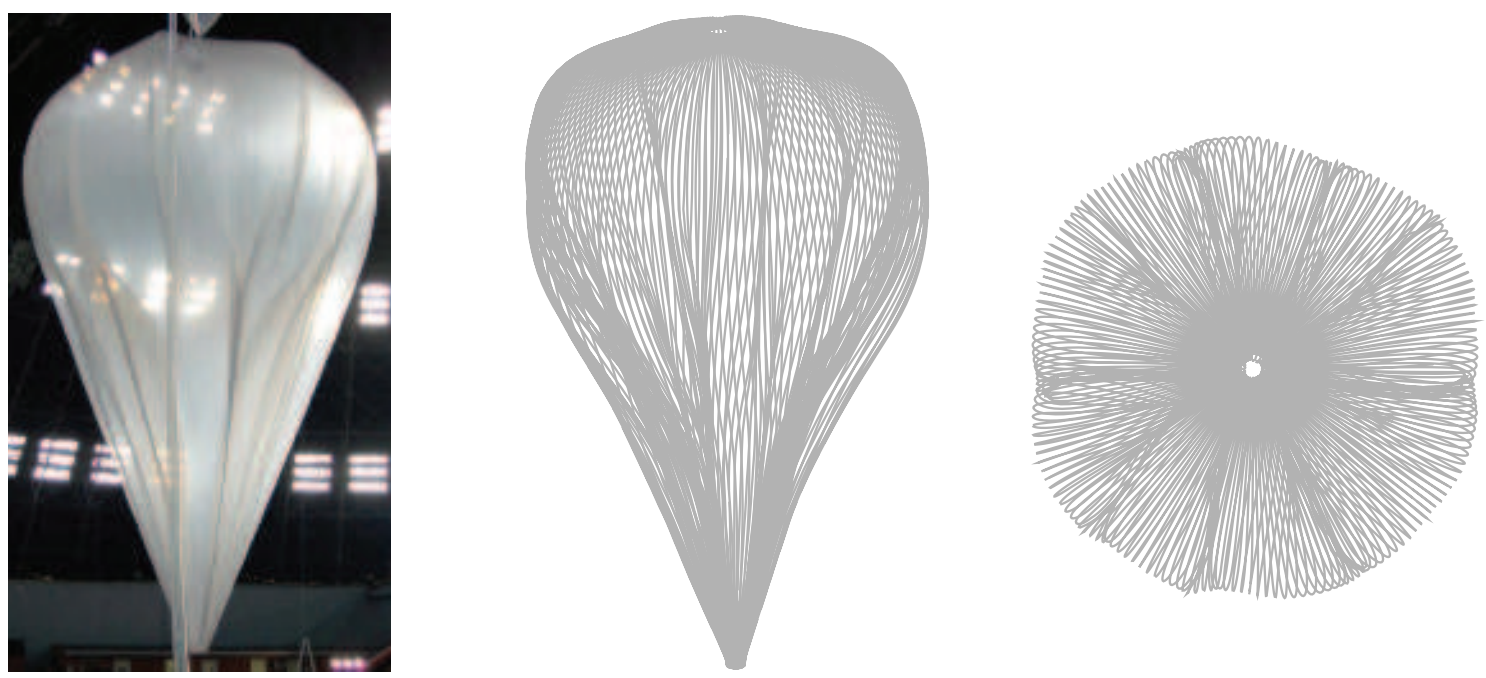

(b) Clefted shape

Figure 15. Shape comparison: (a) initial deployed shape and (b) clefted shape.

\section{6 of 18}


model, an axi-symmetric balloon with no hoop stress is assumed and a finite difference method is applied to solve the resulting differential equation. For a given distribution of differential pressure, an iterative algorithm is implemented to compute its profile. The three-dimensional solutions are obtained with ABAQUS/Explicit, with a user-defined material subroutine that implements a mixed stress-strain wrinkling criterion. Frictionless contact is defined for the surface of balloon to prevent the penetration of the balloon through itself. The initial geometry of the lobes is mapped onto their (flat) cutting pattern, and this allows us to compute the initial stresses and strains with ease.

A study of the shapes taken up by a flat facet ULDB balloon has shown that a region of tensile hoop stress remains in the crown region until the bottom pressure is negative. This region prevents the formation of clefts. Conversely, the formation of clefts requires the disappearance of this stabilizing "tension ring" at the top of the balloon.

It is important to note that none of this is predicted by the two-dimensional, axisymmetric solution. A more detailed comparison between the two solution techniques and the experimental results is presented in Figure 16. The results from ABAQUS/Explicit agree quite well with the Experiment, but there are large differences with the results from the two-dimensional theory.

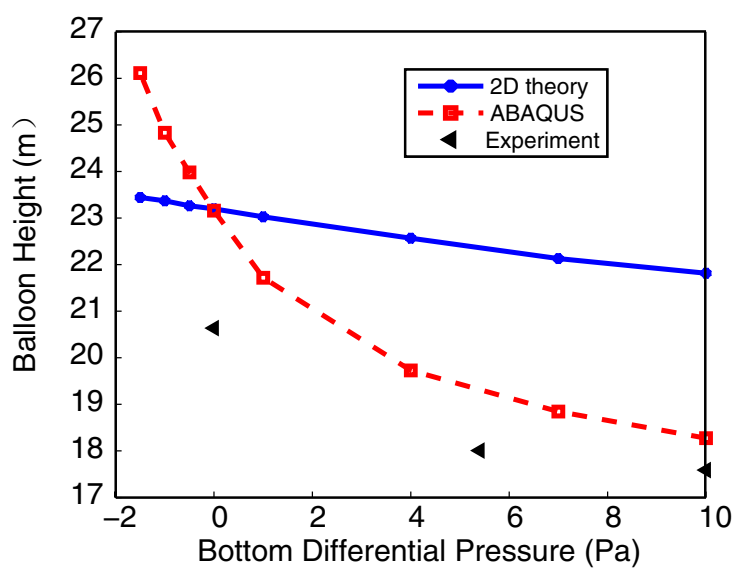

(a)

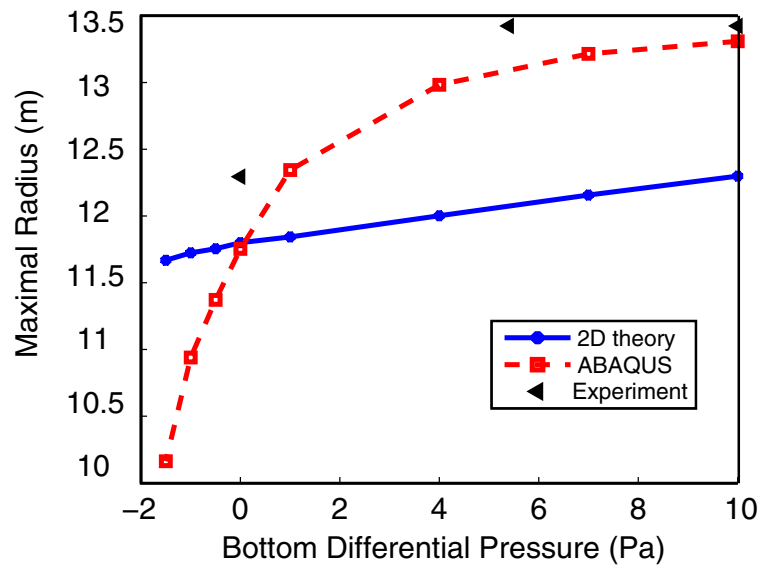

(b)

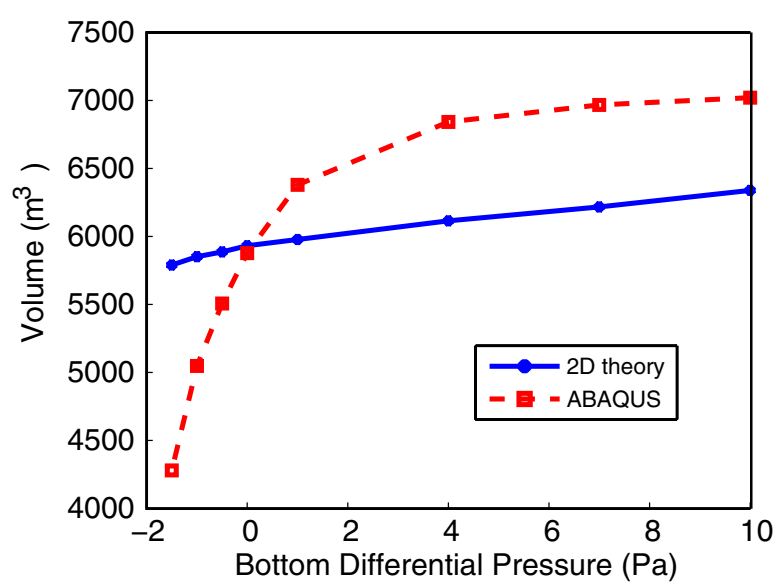

(c)

Figure 16. Shape comparison: (a) bottom differential pressure vs. height relationship and (b) bottom differential pressure vs. maximum radius (to membrane) relationship.

Lastly, it is noted that the present results were obtained for a flat-lobe design; the case of more lobed balloon designs should also be investigated. 


\section{Acknowledgments}

We thank the NASA ULDB team for their contribution to this research. In particular, we are grateful to Henry Cathey for kindly providing test data and Rodger Farley, Jim Rand and David Wakefield for helping us through numerous discussions. This research was supported by the NASA Balloon Program Office (contract monitor Danny Ball).

\section{References}

${ }^{1}$ Cathey, H. M., "Test flights of the NASA ultra-long duration balloon", Advances in Space Research, 33, 1633-1641, 2004.

${ }^{2}$ Nott, J., "Design considerations and practical results with long duration systems for manned world flights", Advances in Space Research, 33, 1667-1673, 2004

${ }^{3}$ Smalley, J. H., "Determination of the shape of a free balloon," Scientific report no. 2, afrl 64-374, Air Force Cambridge Research Labovatories, Bedford, MA, Dec. 1963

${ }^{4}$ Nishimura, J., Okai, S. and Ogita, N. (). "On the natural shape of the plastic balloon", International Symposium on Space Technology and Science, 5, 446-450, 1963.

${ }^{5}$ Rand, J. L., "Shape and stress of an ascending balloon," Report no. wii-9936-fr-01, NASA/Jet Propustion Lab, Pasadena, CA, 26 June 1987

${ }^{6}$ Baginski, F. and Collier, W., "Energy minimizing shapes of patially inflated large scientific baloons," Advances in Space Research, 21, 975-978, 1998.

${ }^{7}$ F. Baginski, Q. C. and Waldman, I., "Designing the shape of a large scientific balloon," Appplied Mathematical Modeling, 25, 953-966, 2001.

${ }^{8}$ Baginski, F., "A mathematical model for a partially inflated balloon with periodic lobes," Advances in Space Research, 30, 1167-1171, 2002

${ }^{9}$ Taylor, G.I., "On the shapes of parachutes". From: The Scientific Papers of Sir G.I. Taylor, edited by G.K. Batchelor, Cambridge University Press, 3, 26-37, 1963.

${ }^{10}$ Calladine, C.R., Stability of the Endeavour balloon. In: Buckling of structures: theory and experiment, edited by I. Elishakoff, J. Arbocz, C. D. Babcock and A. Libai, Elsevier Science Publishers, 133-149, 1988.

${ }^{11}$ Pagitz, M. and Pellegrino, S., "Buckling pressure of "pumpkin" balloons", International Journal of Solids and Structures 44, 6963-6986, 2007.

${ }^{12}$ Lennon, B. A., Equilibrium of Stability of Inflatable Membrane Structures, Ph.D. thesis, Department of Engineering, University of Cambridge, 2002.

${ }^{13}$ Irvine, H., Cable structure, MIT Press, 1981.

${ }^{14}$ Miller, R. K. and Hedgepeth, J. M., "An Algorithm for Finite Element Analysis of Partly Wrinkled Membranes," AIAA Journal, Vol. 20, Dec. 1982, pp. 1761-1763.

${ }^{15}$ Adler, A. L., Finite element approaches for static and dynamic analysis of partially wrinkled membrane structures, Ph.D. thesis, University of Colorado at Boulder, 2000.

${ }^{16}$ Cathey, H. M., "ULDB $27 \mathrm{~m}$ flat facet balloon deployment and pressurization test," Test report, Physical Science Laboratory, NASA BPO, Dec. 2007.

${ }^{17}$ Deng, X., Simulation of partially-filled stratosphere balloon, First year report, Department of Engineering, University of Cambridge, 2007. 